\section{ONOMÁVEIN}

Revista semestral de lingüística, filología y traducción
PONTIFICIA UNIVERSIDAD

CATÓLICA DE CHILE

CATOLICA DE CHILE
FACULTAD DE LETRAS

\title{
GLOSARIO / GLOSSARY
}

\section{Glosario inglés-español: términos en TCL y LSF}

\author{
Beatriz Quiroz \\ Pontificia Universidad Católica de Chile \\ Chile
}

Número especial
ONOMÁZEIN Número especial LSF y TCL sobre educación y conocimiento (2017): 227-242 DOI: 10.7764/onomazein.sfl.og

\section{(C) (i) $\odot$}

Beatriz Quiroz: Departamento de Ciencias del Lenguaje, Facultad de Letras, Pontificia Universidad Católica de Chile, Chile. I Correo electrónico: bquiroz@uc.cl 
A continuación se presenta un glosario bilingüe que abarca la terminología empleada en los artículos de este número especial. De izquierda a derecha, aparece primero una columna con el término original en inglés, luego una columna con el término equivalente en español y, por último, una tercera columna con algunas palabras claves que permiten situar teóricamente el concepto asociado con mayor facilidad. En algunos casos, en esta última columna se proporciona la fuente del concepto y(o) término en cuestión (véase lista de obras citadas al final de este documento). Nótese que los autores que aparecen seguidos de asterisco son tratados en profundidad en los artículos que conforman este mismo número especial. Por último, se presenta una breve lista de las siglas y las abreviaturas empleadas en este documento.

Para la definición de varios de los conceptos de la Teoría de Códigos de Legitimación (TCL) y sus convenciones grafológicas asociadas, véase Maton (2014), Maton, Hood y Shay (2016) y http://www.legitimationcodetheory.com. Para el detalle de las convenciones grafológicas en Lingüística Sistémico Funcional (LSF), véase Quiroz (2016).

\begin{tabular}{|c|c|c|}
\hline TÉRMINO EN INGLÉS & $\begin{array}{l}\text { TRADUCCIÓN AL } \\
\text { ESPAÑOL }\end{array}$ & $\begin{array}{l}\text { CONCEPTOS ASOCIADOS/FUENTES } \\
\text { ASOCIADAS (EN INGLÉS) }\end{array}$ \\
\hline activity & actividad & $\begin{array}{l}\text { SFL, discourse semantics, IDEATION; } \\
\text { Hao (2015) }\end{array}$ \\
\hline AFFECT & AFECTO & SFL, discourse semantics, APPRAISAL \\
\hline aggregation & agregación & SFL, type of mass, textual metafunction \\
\hline APPRAISAL & VALORACIÓN & $\begin{array}{l}\text { SFL, discourse semantics, interpersonal } \\
\text { metafunction }\end{array}$ \\
\hline APPRECIATION & APRECIACIÓN & SFL, discourse semantics, APPRAISAL \\
\hline attitude & actitud & SFL, discourse semantics, APPRAISAL \\
\hline attribution & $\begin{array}{l}\text { (sust.) atribución; (vb.) } \\
\text { atribuir }\end{array}$ & SFL, discourse semantics, APPRAISAL \\
\hline augmenting & $\begin{array}{l}\text { (sust.) incrementamiento; } \\
\text { (vb.) incrementar }\end{array}$ & $\begin{array}{l}\text { LCT, Semantics, semantic density, } \\
\text { degree of epistemological } \\
\text { condensation, clausing analysis }\end{array}$ \\
\hline Autonomy & Autonomía & LCT, dimension \\
\hline axial relation & relación axial & SFL, axis, system network \\
\hline axiological condensation & condensación axiológica & LCT, Semantics, semantic density \\
\hline $\begin{array}{l}\text { axiological-semantic } \\
\text { density }\end{array}$ & $\begin{array}{l}\text { densidad semántica } \\
\text { axiológica }\end{array}$ & LCT, Semantics, semantic density \\
\hline $\begin{array}{l}\text { axiologically charged } \\
\text { discourse }\end{array}$ & $\begin{array}{l}\text { discurso cargado } \\
\text { axiológicamente }\end{array}$ & SFL, discourse semantics, APPRAISAL \\
\hline basis (of practices) & base (de las prácticas) & LCT \\
\hline
\end{tabular}




\begin{tabular}{|c|c|c|}
\hline TÉRMINO EN INGLÉS & $\begin{array}{l}\text { TRADUCCIÓN AL } \\
\text { ESPAÑOL }\end{array}$ & $\begin{array}{l}\text { CONCEPTOS ASOCIADOS/FUENTES } \\
\text { ASOCIADAS (EN INGLÉS) }\end{array}$ \\
\hline Bid move & $\begin{array}{l}\text { movimiento de Solicitud } \\
\text { de turno }\end{array}$ & $\begin{array}{l}\text { SFL, discourse semantics, exchange } \\
\text { structure, R2L; Martin (1992) }\end{array}$ \\
\hline $\begin{array}{l}\text { bonding icons } \\
\text { (bondicons) }\end{array}$ & iconos vinculadores & SFL, instantiation, bonding \\
\hline $\begin{array}{l}\text { categorized } \\
\text { (modification) }\end{array}$ & $\begin{array}{l}\text { modificación por } \\
\text { categorización }\end{array}$ & $\begin{array}{l}\text { LCT, Semantics, semantic density, ESD, } \\
\text { word-grouping }\end{array}$ \\
\hline causing & $\begin{array}{l}\text { (sust.) causación; (vb.) } \\
\text { causar }\end{array}$ & $\begin{array}{l}\text { LCT, Semantics, semantic density, } \\
\text { degree of epistemological } \\
\text { condensation, clausing analysis }\end{array}$ \\
\hline challenging move & $\begin{array}{l}\text { movimiento (interactivo) } \\
\text { de desafío }\end{array}$ & $\begin{array}{l}\text { SFL, discourse semantics, NEGOTIATION, } \\
\text { mass, aggregation; Martin (1992) }\end{array}$ \\
\hline characterizing & $\begin{array}{l}\text { (sust.) caracterización; } \\
\text { (vb.) caracterizar }\end{array}$ & $\begin{array}{l}\text { LCT, Semantics, semantic density, } \\
\text { degree of epistemological } \\
\text { condensation, clausing analysis }\end{array}$ \\
\hline classification relation & relación de clasificación & $\begin{array}{l}\text { SFL, discourse semantics, IDEATION, } \\
\text { taxonomic relations }\end{array}$ \\
\hline classification (taxonomy) & $\begin{array}{l}\text { taxonomía de } \\
\text { clasificación }\end{array}$ & $\begin{array}{l}\text { SFL, discourse semantics, IDEATION, } \\
\text { taxonomic relations }\end{array}$ \\
\hline Classifier & $\begin{array}{l}\text { Clasificador (función de } \\
\text { grupo nominal) }\end{array}$ & SFL, lexicogrammar \\
\hline classifying & $\begin{array}{l}\text { (sust.) clasificación; (vb.) } \\
\text { clasificar }\end{array}$ & $\begin{array}{l}\text { LCT, Semantics, semantic density, } \\
\text { degree of epistemological } \\
\text { condensation, clausing analysis }\end{array}$ \\
\hline clausing (tool) & $\begin{array}{l}\text { (sust.) enclausamiento } \\
\text { (, herramienta de); (vb.) } \\
\text { enclausar }\end{array}$ & $\begin{array}{l}\text { LCT, Semantics, semantic density, } \\
\text { degree of epistemological } \\
\text { condensation }\end{array}$ \\
\hline cline & clina & SFL \\
\hline code clash & choque de códigos & LCT \\
\hline code drift & $\begin{array}{l}\text { desplazamiento de } \\
\text { código }\end{array}$ & LCT, movement within quadrants \\
\hline code match & coincidencia de códigos & LCT \\
\hline code shift & cambio de código & LCT, movement across quadrants \\
\hline code theory & teoría de códigos & Bernstein \\
\hline $\begin{array}{l}\text { coherent (segmental) } \\
\text { sequencing }\end{array}$ & $\begin{array}{l}\text { secuenciamiento } \\
\text { (segmental) coherente }\end{array}$ & $\begin{array}{l}\text { LCT, Semantics, semantic density, } \\
\text { degree of epistemological } \\
\text { condensation, sequencing analysis }\end{array}$ \\
\hline
\end{tabular}




\begin{tabular}{|c|c|c|}
\hline TÉRMINO EN INGLÉS & $\begin{array}{l}\text { TRADUCCIÓN AL } \\
\text { ESPAÑOL }\end{array}$ & $\begin{array}{l}\text { CONCEPTOS ASOCIADOS/FUENTES } \\
\text { ASOCIADAS (EN INGLÉS) }\end{array}$ \\
\hline $\begin{array}{l}\text { common (everyday) } \\
\text { words }\end{array}$ & $\begin{array}{l}\text { palabra (cotidiana) } \\
\text { común }\end{array}$ & $\begin{array}{l}\text { LCT, Semantics, semantic density, ESD, } \\
\text { wording tool }\end{array}$ \\
\hline common sense & (de) sentido común & Bernstein, SFL \\
\hline compact (technical) word & $\begin{array}{l}\text { palabra (técnica) } \\
\text { compacta }\end{array}$ & $\begin{array}{l}\text { LCT, Semantics, semantic density, ESD, } \\
\text { wording tool }\end{array}$ \\
\hline compact-element & $\begin{array}{l}\text { palabra compacta de } \\
\text { elemento }\end{array}$ & $\begin{array}{l}\text { LCT, Semantics, semantic density, ESD, } \\
\text { wording tool, technicals }\end{array}$ \\
\hline compact-property & $\begin{array}{l}\text { palabra compacta de } \\
\text { propiedad }\end{array}$ & $\begin{array}{l}\text { LCT, Semantics, semantic density, ESD, } \\
\text { wording tool, technicals }\end{array}$ \\
\hline $\begin{array}{l}\text { compartmental } \\
\text { (segmental) sequencing }\end{array}$ & $\begin{array}{l}\text { secuenciamiento } \\
\text { (segmental) } \\
\text { compartimental }\end{array}$ & $\begin{array}{l}\text { LCT, Semantics, semantic density, } \\
\text { degree of epistemological } \\
\text { condensation, sequencing analysis }\end{array}$ \\
\hline composing (n) & composición & $\begin{array}{l}\text { LCT, Semantics, semantic density, } \\
\text { degree of epistemological } \\
\text { condensation, clausing analysis }\end{array}$ \\
\hline composition (taxonomy) & $\begin{array}{l}\text { taxonomía de } \\
\text { composición }\end{array}$ & $\begin{array}{l}\text { SFL, discourse semantics, IDEATION, } \\
\text { taxonomic relations }\end{array}$ \\
\hline compositional relation & relación de composición & $\begin{array}{l}\text { SFL, discourse semantics, IDEATION, } \\
\text { taxonomic relations }\end{array}$ \\
\hline $\begin{array}{l}\text { condensation } \\
\text { (strengthening semantic } \\
\text { density) }\end{array}$ & $\begin{array}{l}\text { condensación } \\
\text { (fortalecimiento de } \\
\text { densidad semántica) }\end{array}$ & LCT, Semantics, semantic density \\
\hline $\begin{array}{l}\text { conglomerate (technical) } \\
\text { word }\end{array}$ & $\begin{array}{l}\text { palabra (técnica) } \\
\text { conglomerada }\end{array}$ & $\begin{array}{l}\text { LCT, Semantics, semantic density, ESD, } \\
\text { wording tool }\end{array}$ \\
\hline conglomerate-element & $\begin{array}{l}\text { palabra conglomerada de } \\
\text { elemento }\end{array}$ & $\begin{array}{l}\text { LCT, Semantics, semantic density, ESD, } \\
\text { wording tool, technicals }\end{array}$ \\
\hline conglomerate-property & $\begin{array}{l}\text { palabra conglomerada de } \\
\text { propiedad }\end{array}$ & $\begin{array}{l}\text { LCT, Semantics, semantic density, ESD, } \\
\text { wording tool, technicals }\end{array}$ \\
\hline CONJUNCTION & CONJUNCIÓN & $\begin{array}{l}\text { SFL, discourse semantics, } \\
\text { CONJUNCTION, ideational metafunction }\end{array}$ \\
\hline connecting & $\begin{array}{l}\text { (sust.) enlazamiento; (vb.) } \\
\text { enlazar }\end{array}$ & $\begin{array}{l}\text { LCT, Semantics, semantic density, } \\
\text { degree of epistemological } \\
\text { condensation, clausing analysis }\end{array}$ \\
\hline $\begin{array}{l}\text { consequential } \\
\text { (cumulative) sequencing }\end{array}$ & $\begin{array}{l}\text { secuenciamiento } \\
\text { (acumulativo) } \\
\text { consecutivo }\end{array}$ & $\begin{array}{l}\text { LCT, Semantics, semantic density, } \\
\text { degree of epistemological } \\
\text { condensation, sequencing analysis }\end{array}$ \\
\hline consolidated word & palabra consolidada & $\begin{array}{l}\text { LCT, Semantics, semantic density, ESD, } \\
\text { wording tool, everydays }\end{array}$ \\
\hline
\end{tabular}




\begin{tabular}{|c|c|c|}
\hline TÉRMINO EN INGLÉS & $\begin{array}{l}\text { TRADUCCIÓN AL } \\
\text { ESPAÑOL }\end{array}$ & $\begin{array}{l}\text { CONCEPTOS ASOCIADOS/FUENTES } \\
\text { ASOCIADAS (EN INGLÉS) }\end{array}$ \\
\hline constellation & constelación & LCT \\
\hline coordinating & $\begin{array}{l}\text { (sust.) vinculación; (vb.) } \\
\text { vincular }\end{array}$ & $\begin{array}{l}\text { LCT, Semantics, semantic density, } \\
\text { degree of epistemological } \\
\text { condensation, clausing analysis }\end{array}$ \\
\hline correlating & $\begin{array}{l}\text { (sust.) correlación; (vb.) } \\
\text { correlacionar }\end{array}$ & $\begin{array}{l}\text { LCT, Semantics, semantic density, } \\
\text { degree of epistemological } \\
\text { condensation, clausing analysis }\end{array}$ \\
\hline coupling & $\begin{array}{l}\text { (sust.) acoplamiento; (vb.) } \\
\text { acoplar }\end{array}$ & SFL, Martin, instantiation \\
\hline cumulative sequencing & $\begin{array}{l}\text { secuenciamiento } \\
\text { acumulativo }\end{array}$ & $\begin{array}{l}\text { LCT, Semantics, semantic density, } \\
\text { degree of epistemological } \\
\text { condensation, sequencing analysis }\end{array}$ \\
\hline $\begin{array}{l}\text { degrees of } \\
\text { epistemological } \\
\text { condensation }\end{array}$ & $\begin{array}{l}\text { grados de condensación } \\
\text { epistemológica }\end{array}$ & LCT, Semantics, ESD \\
\hline $\begin{array}{l}\text { delayed primary knower } \\
\text { (dK1) }\end{array}$ & $\begin{array}{l}\text { conocedor primario } \\
\text { diferido (CId) }\end{array}$ & $\begin{array}{l}\text { SFL, discourse semantics, NEGOTIATION, } \\
\text { exchange structure }\end{array}$ \\
\hline delicacy & delicadeza & SFL \\
\hline delicate & (adj.) delicado & SFL, systems, delicacy \\
\hline descriptive phases & fases descriptivas & SFL; Hood (este número especial) \\
\hline differentiation & diferenciación & LCT, Semantics, semantic density, ESD \\
\hline $\begin{array}{l}\text { dimension, } \\
\text { dimensionality }\end{array}$ & $\begin{array}{l}\text { dimensión, } \\
\text { dimensionalidad }\end{array}$ & $\begin{array}{l}\text { SFL, discourse semantics, IDEATION; } \\
\text { Hao (2015) }\end{array}$ \\
\hline displaying & $\begin{array}{l}\text { (sust.) despliegue; (vb.) } \\
\text { desplegar }\end{array}$ & $\begin{array}{l}\text { LCT, Semantics, semantic density, } \\
\text { degree of epistemological } \\
\text { condensation, clausing analysis }\end{array}$ \\
\hline doxa & doxa & SFL, instantiation, bonding; Tann (2012) \\
\hline dramatizing & $\begin{array}{l}\text { (sust.) dramatización; (vb.) } \\
\text { dramatizar }\end{array}$ & $\begin{array}{l}\text { LCT, Semantics, semantic density, } \\
\text { degree of epistemological } \\
\text { condensation, clausing analysis }\end{array}$ \\
\hline Elaborate (phase) & Elaborar (, fase de) & $S F L, R 2 L$ \\
\hline élite code & código de elite & LCT, Specialization \\
\hline embedded (modification) & $\begin{array}{l}\text { (modificación por) } \\
\text { incrustación }\end{array}$ & $\begin{array}{l}\text { LCT, Semantics, semantic density, ESD, } \\
\text { word-grouping }\end{array}$ \\
\hline ENGAGEMENT & COMPROMISO & SFL, discourse semantics, APPRAISAL \\
\hline
\end{tabular}




\begin{tabular}{|c|c|c|}
\hline TÉRMINO EN INGLÉS & $\begin{array}{l}\text { TRADUCCIÓN AL } \\
\text { ESPAÑOL }\end{array}$ & $\begin{array}{l}\text { CONCEPTOS ASOCIADOS/FUENTES } \\
\text { ASOCIADAS (EN INGLÉS) }\end{array}$ \\
\hline entity & entidad & $\begin{array}{l}\text { SFL, discourse semantics, IDEATION; } \\
\text { Hao (2015) }\end{array}$ \\
\hline epistemic relations (ER) & $\begin{array}{l}\text { relaciones epistémicas } \\
(\mathrm{RE})\end{array}$ & LCT, Specialization \\
\hline $\begin{array}{l}\text { epistemic-semantic } \\
\text { density (ESD) }\end{array}$ & $\begin{array}{l}\text { densidad semántica } \\
\text { epistémica (DSE) }\end{array}$ & LCT, Semantics, semantic density \\
\hline $\begin{array}{l}\text { epistemological } \\
\text { condensation (EC) }\end{array}$ & $\begin{array}{l}\text { condensación } \\
\text { epistemológica (CE) }\end{array}$ & LCT, Semantics, semantic density \\
\hline $\begin{array}{l}\text { epistemological } \\
\text { rarefaction }\end{array}$ & $\begin{array}{l}\text { rarificación } \\
\text { epistemológica }\end{array}$ & LCT, Semantics, semantic density \\
\hline equating & $\begin{array}{l}\text { (sust.) equiparación; (vb.) } \\
\text { equiparar }\end{array}$ & $\begin{array}{l}\text { LCT, Semantics, semantic density, } \\
\text { degree of epistemological } \\
\text { condensation, clausing analysis }\end{array}$ \\
\hline ESD profile & $\begin{array}{l}\text { perfil de DSE (densidad } \\
\text { semántica epistémica) }\end{array}$ & LCT, Semantics, semantic density \\
\hline ESD strength & $\begin{array}{l}\text { fortaleza de DSE } \\
\text { (densidad semántica } \\
\text { epistémica) }\end{array}$ & LCT, Semantics, semantic density \\
\hline establishing & $\begin{array}{l}\text { (sust.) establecimiento; } \\
\text { (vb.) establecer }\end{array}$ & $\begin{array}{l}\text { LCT, Semantics, semantic density, } \\
\text { degree of epistemological } \\
\text { condensation, clausing analysis }\end{array}$ \\
\hline Evaluate (phase) & Evaluar (, fase de) & $\mathrm{SFL}, \mathrm{R} 2 \mathrm{~L}$ \\
\hline everyday word & palabra cotidiana & $\begin{array}{l}\text { LCT, Semantics, semantic density, ESD, } \\
\text { wording tool }\end{array}$ \\
\hline exchange structure & $\begin{array}{l}\text { estructura de } \\
\text { intercambio }\end{array}$ & $\begin{array}{l}\text { SFL, discourse semantics, NEGOTIATION, } \\
\text { exchange structure }\end{array}$ \\
\hline explaining phase & fase de explicación & SFL; Hood (este número especial) \\
\hline external language & lenguaje externo & Bernstein, LCT \\
\hline feature & rasgo (sistémico) & SFL, axis, system network \\
\hline field & campo & SFL \\
\hline flatline & perfil plano & LCT \\
\hline flexi-tech & $\begin{array}{l}\text { flexi-tecnicalidad, } \\
\text { términos flexi-técnicos }\end{array}$ & SFL, humanities discourse \\
\hline focus (of practices) & foco (de las prácticas) & LCT \\
\hline Focus (phase) & Focalizar (, fase de) & $\mathrm{SFL}, \mathrm{R} 2 \mathrm{~L}$ \\
\hline
\end{tabular}




\begin{tabular}{|c|c|c|}
\hline TÉRMINO EN INGLÉS & $\begin{array}{l}\text { TRADUCCIÓN AL } \\
\text { ESPAÑOL }\end{array}$ & $\begin{array}{l}\text { CONCEPTOS ASOCIADOS/FUENTES } \\
\text { ASOCIADAS (EN INGLÉS) }\end{array}$ \\
\hline gaze & mirada & LCT, Specialization \\
\hline gemeinschaft & gemeinschaft & SFL, instantiation, bonding; Tann (2012) \\
\hline $\begin{array}{l}\text { generalist (consolidated) } \\
\text { word }\end{array}$ & $\begin{array}{l}\text { palabra (consolidada) } \\
\text { generalista }\end{array}$ & $\begin{array}{l}\text { LCT, Semantics, semantic density, ESD, } \\
\text { wording tool, everydays }\end{array}$ \\
\hline generality & generalidad & LCT, Semantics, semantic density \\
\hline genre & género & SFL, highest stratum \\
\hline grammatical metaphor & metáfora gramatical & SFL \\
\hline grammaticality & gramaticalidad & Bernstein*, Muller (2007) \\
\hline $\begin{array}{l}\text { hierarchical knower } \\
\text { structure }\end{array}$ & $\begin{array}{l}\text { estructura de conocedor } \\
\text { jerárquica }\end{array}$ & LCT \\
\hline $\begin{array}{l}\text { hierarchical knowledge } \\
\text { structure }\end{array}$ & $\begin{array}{l}\text { estructura de } \\
\text { conocimiento jerárquica }\end{array}$ & Bernstein, LCT \\
\hline hierarchy of instantiation & $\begin{array}{l}\text { jerarquía de } \\
\text { instanciación }\end{array}$ & SFL \\
\hline high flatline & perfil plano elevado & LCT \\
\hline high level periodicity & $\begin{array}{l}\text { periodicidad de nivel(es) } \\
\text { superior(es) }\end{array}$ & SFL, discourse semantics, PERIODICITY \\
\hline $\begin{array}{l}\text { higher/lower } \\
\text { epistemological } \\
\text { condensation (EC+/-) }\end{array}$ & $\begin{array}{l}\text { condensación } \\
\text { epistemológica mayor/ } \\
\text { menor (CE+/ᄀ-) }\end{array}$ & $\begin{array}{l}\text { LCT, Semantics, semantic density, } \\
\text { change in ESD }\end{array}$ \\
\hline higher level New & Nuevo de nivel superior & $\begin{array}{l}\text { SFL, discourse semantics, PERIODICITY, } \\
\text { mass, aggregation }\end{array}$ \\
\hline higher level Theme & Tema de nivel superior & $\begin{array}{l}\text { SFL, discourse semantics, PERIODICITY, } \\
\text { mass, aggregation }\end{array}$ \\
\hline historical recount & recuento histórico & SFL, genre type \\
\hline $\begin{array}{l}\text { horizontal (cumulative) } \\
\text { sequencing }\end{array}$ & $\begin{array}{l}\text { secuenciamiento } \\
\text { (acumulativo) horizontal }\end{array}$ & $\begin{array}{l}\text { LCT, Semantics, semantic density, } \\
\text { degree of epistemological } \\
\text { condensation, sequencing analysis }\end{array}$ \\
\hline horizontal discourse & discurso horizontal & Bernstein* \\
\hline $\begin{array}{l}\text { horizontal knower } \\
\text { structure }\end{array}$ & $\begin{array}{l}\text { estructura de conocedor } \\
\text { horizontal }\end{array}$ & LCT \\
\hline $\begin{array}{l}\text { horizontal knowledge } \\
\text { structure }\end{array}$ & $\begin{array}{l}\text { estructura de } \\
\text { conocimiento horizontal }\end{array}$ & Bernstein*, LCT \\
\hline Hyper-New & Hiper-Nuevo & $\begin{array}{l}\text { SFL, discourse semantics, PERIODICITY, } \\
\text { mass, aggregation }\end{array}$ \\
\hline
\end{tabular}




\begin{tabular}{|c|c|c|}
\hline TÉRMINO EN INGLÉS & $\begin{array}{l}\text { TRADUCCIÓN AL } \\
\text { ESPAÑOL }\end{array}$ & $\begin{array}{l}\text { CONCEPTOS ASOCIADOS/FUENTES } \\
\text { ASOCIADAS (EN INGLÉS) }\end{array}$ \\
\hline hyper-technicality & hipertecnicalidad & SFL; Doran (2010) \\
\hline Hyper-Theme & Hiper-Tema & $\begin{array}{l}\text { SFL, discourse semantics, PERIODICITY, } \\
\text { mass, aggregation }\end{array}$ \\
\hline iconicity & iconicidad & $\begin{array}{l}\text { SFL, ideational metafunction, type of } \\
\text { presence }\end{array}$ \\
\hline iconization & iconización & $\begin{array}{l}\text { SFL, type of mass, interpersonal } \\
\text { metafunction }\end{array}$ \\
\hline IDEATION & IDEACIÓN & SFL, discourse semantics \\
\hline IDENTIFICATION & IDENTIFICACIÓN & $\begin{array}{l}\text { SFL, discourse semantics, ideational } \\
\text { metafunction. }\end{array}$ \\
\hline implication complex & complejo de implicación & SFL; Doran (2015) \\
\hline implication sequence & secuencia de implicación & $\begin{array}{l}\text { SFL, discourse semantics, IDEATION, } \\
\text { activity sequences }\end{array}$ \\
\hline implicitness & implicitud & $\begin{array}{l}\text { SFL, textual metafunction, type of } \\
\text { presence }\end{array}$ \\
\hline $\begin{array}{l}\text { incoherent (segmental) } \\
\text { sequencing }\end{array}$ & $\begin{array}{l}\text { secuenciamiento } \\
\text { (segmental) incoherente }\end{array}$ & $\begin{array}{l}\text { LCT, Semantics, semantic density, } \\
\text { degree of epistemological } \\
\text { condensation, sequencing analysis }\end{array}$ \\
\hline individuation/affiliation & individuación-afiliación & SFL; Martin* \\
\hline instantiation & instanciación & SFL \\
\hline $\begin{array}{l}\text { integrative (cumulative) } \\
\text { sequencing }\end{array}$ & $\begin{array}{l}\text { secuenciamiento } \\
\text { (acumulativo) integrativo }\end{array}$ & $\begin{array}{l}\text { LCT, Semantics, semantic density, } \\
\text { degree of epistemological } \\
\text { condensation, sequencing analysis }\end{array}$ \\
\hline intellectual field & campo intelectual & LCT \\
\hline internal conjunction & conjunción interna & SFL, discourse semantics, CONJUNCTION \\
\hline $\begin{array}{l}\text { interpersonal } \\
\text { aggregation }\end{array}$ & agregación interpersonal & SFL, mass, aggregation \\
\hline invoked attitude & actitud invocada & SFL, discourse semantics, APPRAISAL \\
\hline JUDGEMENT & jUICIO & SFL, discourse semantics, APPRAISAL \\
\hline knower code & código de conocedor & LCT, Specialization \\
\hline knower structure & estructura de conocedor & LCT, Specialization \\
\hline knowledge-building & $\begin{array}{l}\text { (sust.) construcción } \\
\text { de conocimiento; ( vb.) } \\
\text { construir conocimiento }\end{array}$ & LCT \\
\hline
\end{tabular}




\begin{tabular}{|c|c|c|}
\hline TÉRMINO EN INGLÉS & $\begin{array}{l}\text { TRADUCCIÓN AL } \\
\text { ESPAÑOL }\end{array}$ & $\begin{array}{l}\text { CONCEPTOS ASOCIADOS/FUENTES } \\
\text { ASOCIADAS (EN INGLÉS) }\end{array}$ \\
\hline knowledge claim & $\begin{array}{l}\text { afirmación de } \\
\text { conocimiento }\end{array}$ & LCT \\
\hline knowledge code & código de conocimiento & LCT, Specialization \\
\hline knowledge move & $\begin{array}{l}\text { movimiento (interactivo) } \\
\text { de conocimiento }\end{array}$ & SFL, discourse semantics, NEGOTIATION \\
\hline knowledge practices & $\begin{array}{l}\text { prácticas de } \\
\text { conocimiento }\end{array}$ & LCT \\
\hline knowledge structure & $\begin{array}{l}\text { estructura de } \\
\text { conocimiento }\end{array}$ & Bernstein*, LCT \\
\hline $\begin{array}{l}\text { knowledge knower } \\
\text { structures }\end{array}$ & $\begin{array}{l}\text { estructuras de } \\
\text { conocimiento-conocedor }\end{array}$ & LCT, Specialization \\
\hline language of description & lenguaje de descripción & Bernstein*, LCT \\
\hline learning activity & actividad de aprendizaje & $\mathrm{SFL}, \mathrm{R} 2 \mathrm{~L}$ \\
\hline legitimation code & código de legitimación & LCT \\
\hline $\begin{array}{l}\text { Legitimation Code Theory } \\
\text { (LCT) }\end{array}$ & $\begin{array}{l}\text { Teoría de Códigos de } \\
\text { Legitimación (TCL) }\end{array}$ & LCT \\
\hline lesson stage & $\begin{array}{l}\text { etapa de la clase; etapa } \\
\text { de la lección }\end{array}$ & SFL, R2L \\
\hline lexical string & cadena léxica & SFL, IDEATION, taxonomic relations \\
\hline located (modification) & $\begin{array}{l}\text { modificación por } \\
\text { localización }\end{array}$ & $\begin{array}{l}\text { LCT, Semantics, semantic density, ESD, } \\
\text { word-grouping }\end{array}$ \\
\hline logogenetic (text time) & $\begin{array}{l}\text { (tiempo del texto) } \\
\text { logogenético }\end{array}$ & SFL, instantiation, semogenesis \\
\hline Macro-New & Macro-Nuevo & $\begin{array}{l}\text { SFL, discourse semantics, PERIODICITY, } \\
\text { mass, aggregation }\end{array}$ \\
\hline Macro-Theme & Macro-Tema & $\begin{array}{l}\text { SFL, discourse semantics, PERIODICITY, } \\
\text { mass, aggregation }\end{array}$ \\
\hline mass & masa & SFL \\
\hline mediating language & lenguaje mediador & LCT \\
\hline metadiscourse & metadiscurso & SFL \\
\hline modalities & modalidades & LCT, codes, quadrants \\
\hline mode & modo & SFL, register \\
\hline MOOD & MODO & SFL, lexicogrammar, interpersonal \\
\hline multimodal text & texto multimodal & SFL, multimodality, mass, aggregation \\
\hline
\end{tabular}




\begin{tabular}{|c|c|c|}
\hline TÉRMINO EN INGLÉS & $\begin{array}{l}\text { TRADUCCIÓN AL } \\
\text { ESPAÑOL }\end{array}$ & $\begin{array}{l}\text { CONCEPTOS ASOCIADOS/FUENTES } \\
\text { ASOCIADAS (EN INGLÉS) }\end{array}$ \\
\hline negotiability & negociabilidad & $\begin{array}{l}\text { SFL, interpersonal metafunction, type } \\
\text { of presence }\end{array}$ \\
\hline NEGOTIATION & NEGOCIACIÓN & $\begin{array}{l}\text { SFL, discourse semantics, exchange } \\
\text { structure }\end{array}$ \\
\hline nuanced (common) word & $\begin{array}{l}\text { palabra (común) } \\
\text { matizada }\end{array}$ & $\begin{array}{l}\text { LCT, Semantics, semantic density, ESD, } \\
\text { wording tool, everydays }\end{array}$ \\
\hline object of study & objeto de estudio & LCT \\
\hline ontogenetic (life-time) & $\begin{array}{l}\text { (tiempo vital) } \\
\text { ontogenético }\end{array}$ & SFL, instantiation, semogenesis \\
\hline oracle & oráculo & SFL, instantiation, bonding; Tann (2012) \\
\hline $\begin{array}{l}\text { pedagogic exchange } \\
\text { structure }\end{array}$ & $\begin{array}{l}\text { estructura de } \\
\text { intercambio pedagógico }\end{array}$ & SFL, R2L \\
\hline PERIODICITY & PERIODICIDAD & $\begin{array}{l}\text { SFL, discourse semantics, textual } \\
\text { metafunction }\end{array}$ \\
\hline phase (of discourse) & fase (de discurso) & $\begin{array}{l}\text { SFL, discourse semantics, PERIODICITY, } \\
\text { mass, aggregation }\end{array}$ \\
\hline phase shift & cambio de fase & SFL, discourse semantics, genre \\
\hline $\begin{array}{l}\text { phylogenetic } \\
\text { (evolutionary time) }\end{array}$ & $\begin{array}{l}\text { (tiempo evolutivo) } \\
\text { filogenético }\end{array}$ & SFL, instantiation \\
\hline plain (common) word & palabra (común) Ilana & $\begin{array}{l}\text { LCT, Semantics, semantic density, ESD, } \\
\text { wording tool, everydays }\end{array}$ \\
\hline positing & $\begin{array}{l}\text { (sust.) planteamiento; } \\
\text { (vb.) plantear }\end{array}$ & $\begin{array}{l}\text { LCT, Semantics, semantic density, } \\
\text { degree of epistemological } \\
\text { condensation, clausing analysis }\end{array}$ \\
\hline positioning & $\begin{array}{l}\text { (sust.) posicionamiento; } \\
\text { (vb.) posicionar }\end{array}$ & $\begin{array}{l}\text { LCT, Semantics, semantic density, } \\
\text { degree of epistemological } \\
\text { condensation, clausing analysis }\end{array}$ \\
\hline Prepare (phase) & Preparar (, fase de) & SFL, R2L \\
\hline presence & presencia & SFL \\
\hline $\begin{array}{l}\text { primary knower move } \\
\text { (K1) }\end{array}$ & $\begin{array}{l}\text { movimiento de } \\
\text { conocedor primario (Cı) }\end{array}$ & $\begin{array}{l}\text { SFL, discourse semantics, NEGOTIATION, } \\
\text { exchange structure }\end{array}$ \\
\hline proclaim & $\begin{array}{l}\text { (sust.) proclamación; (vb.) } \\
\text { proclamar }\end{array}$ & SFL, discourse semantics, APPRAISAL \\
\hline profiling & $\begin{array}{l}\text { (sust.) generación de } \\
\text { perfil (semántico); (vb.) } \\
\text { generar perfil (semántico) }\end{array}$ & LCT \\
\hline
\end{tabular}




\begin{tabular}{|c|c|c|}
\hline TÉRMINO EN INGLÉS & $\begin{array}{l}\text { TRADUCCIÓN AL } \\
\text { ESPAÑOL }\end{array}$ & $\begin{array}{l}\text { CONCEPTOS ASOCIADOS/FUENTES } \\
\text { ASOCIADAS (EN INGLÉS) }\end{array}$ \\
\hline prosaic code & código prosaico & $\begin{array}{l}\text { LCT, Semantics, semantic gravity, } \\
\text { semantic density }\end{array}$ \\
\hline prosodic & (adj.) prosódico & $\begin{array}{l}\text { SFL, interpersonal metafunction, type } \\
\text { of structure }\end{array}$ \\
\hline proxy word & (palabra) sustitutiva & LCT, Semantics, semantic density, ESD \\
\hline quadrant & cuadrante & LCT \\
\hline quality & cualidad & $\begin{array}{l}\text { SFL, discourse semantics, taxonomic } \\
\text { relations; Hao (2015) }\end{array}$ \\
\hline rarefied code & código rarificado & $\begin{array}{l}\text { LCT, Semantics, semantic gravity, } \\
\text { semantic density }\end{array}$ \\
\hline realization & realización & SFL \\
\hline realization hierarchy & jerarquía de realización & SFL \\
\hline reciprocity of choice & reciprocidad de opción & SFL, tenor; Poynton (1990) \\
\hline register & registro & SFL, register \\
\hline $\begin{array}{l}\text { reiterative (segmental) } \\
\text { sequencing }\end{array}$ & $\begin{array}{l}\text { secuenciamiento } \\
\text { (segmental) reiterativo }\end{array}$ & $\begin{array}{l}\text { LCT, Semantics, semantic density, } \\
\text { degree of epistemological } \\
\text { condensation, sequencing analysis }\end{array}$ \\
\hline relationality & relacionalidad & LCT, Semantics, semantic density, ESD \\
\hline relative strength & fortaleza relativa & LCT \\
\hline relativist code & código relativista & LCT, Specialization \\
\hline $\begin{array}{l}\text { repetitive (segmental) } \\
\text { sequencing }\end{array}$ & $\begin{array}{l}\text { secuenciamiento } \\
\text { (segmental) repetitivo }\end{array}$ & $\begin{array}{l}\text { LCT, Semantics, semantic density, } \\
\text { degree of epistemological } \\
\text { condensation, sequencing analysis }\end{array}$ \\
\hline reporting phases & fases de reporte & SFL; Hood (este número especial) \\
\hline resonance & resonancia & LCT, Semantics, semantic density, ESD \\
\hline rhizomatic code & código rizomático & $\begin{array}{l}\text { LCT, Semantics, semantic gravity, } \\
\text { semantic density }\end{array}$ \\
\hline scaffolding & $\begin{array}{l}\text { (sust.) andamiaje; (vb.) } \\
\text { andamiar }\end{array}$ & $\begin{array}{l}\text { SFL, discourse semantics, PERIODICITY, } \\
\text { internal conjunction, text reference, } \\
\text { metadiscourse, R2L }\end{array}$ \\
\hline $\begin{array}{l}\text { secondary knower move } \\
\text { (K2) }\end{array}$ & $\begin{array}{l}\text { movimiento de } \\
\text { conocedor secundario } \\
\text { (C2) }\end{array}$ & $\begin{array}{l}\text { SFL, discourse semantics, NEGOTIATION, } \\
\text { exchange structure }\end{array}$ \\
\hline
\end{tabular}




\begin{tabular}{|c|c|c|}
\hline TÉRMINO EN INGLÉS & $\begin{array}{l}\text { TRADUCCIÓN AL } \\
\text { ESPAÑOL }\end{array}$ & $\begin{array}{l}\text { CONCEPTOS ASOCIADOS/FUENTES } \\
\text { ASOCIADAS (EN INGLÉS) }\end{array}$ \\
\hline $\begin{array}{l}\text { sedimental (segmental) } \\
\text { sequencing }\end{array}$ & $\begin{array}{l}\text { secuenciamiento } \\
\text { (segmental) sedimental }\end{array}$ & $\begin{array}{l}\text { LCT, Semantics, semantic density, } \\
\text { degree of epistemological } \\
\text { condensation, sequencing analysis }\end{array}$ \\
\hline segmental sequencing & $\begin{array}{l}\text { secuenciamiento } \\
\text { segmental }\end{array}$ & $\begin{array}{l}\text { LCT, Semantics, semantic density, } \\
\text { degree of epistemological } \\
\text { condensation, sequencing analysis }\end{array}$ \\
\hline semantic code & código semántico & LCT, Semantics \\
\hline semantic density (SD) & densidad semántica (DS) & LCT, Semantics \\
\hline semantic flatline & perfil semántico plano & LCT, Semantics \\
\hline semantic gravity (SG) & gravedad semántica (GS) & LCT, Semantics \\
\hline semantic plane & plano semántico & LCT, Semantics \\
\hline semantic profile & perfil semántico & LCT, Semantics \\
\hline semantic range & $\begin{array}{l}\text { rango de variación } \\
\text { semántica }\end{array}$ & LCT, Semantics \\
\hline semantic structure & estructura semántica & LCT, Semantics \\
\hline semantic wave & ondulación semántica & LCT, Semantics \\
\hline Semantics & Semántica & LCT, dimension \\
\hline sequence & secuencia & $\begin{array}{l}\text { SFL, discourse semantics; Halliday y } \\
\text { Matthiessen (1999), Hao (2015) }\end{array}$ \\
\hline $\begin{array}{l}\text { sequence of activity, } \\
\text { activity sequence }\end{array}$ & secuencia de actividades & SFL; Martin (1992) \\
\hline sequencing (tool) & $\begin{array}{l}\text { (sust.) secuenciamiento } \\
\text { (, herramienta de); (vb.) } \\
\text { secuenciar }\end{array}$ & LCT, Semantics, semantic density, ESD \\
\hline $\begin{array}{l}\text { sequential (cumulative) } \\
\text { sequencing }\end{array}$ & $\begin{array}{l}\text { secuenciamiento } \\
\text { (acumulativo) secuencial }\end{array}$ & $\begin{array}{l}\text { LCT, Semantics, semantic density, } \\
\text { degree of epistemological } \\
\text { condensation, sequencing analysis }\end{array}$ \\
\hline shift in register & cambio en el registro & SFL, register \\
\hline social field & campo social ${ }^{1}$ & LCT \\
\hline social realism & realismo social & sociology of education \\
\hline
\end{tabular}

1 No confundir con 'campo' (field) como variable de registro en la LSF. 


\begin{tabular}{|c|c|c|}
\hline TÉRMINO EN INGLÉS & $\begin{array}{l}\text { TRADUCCIÓN AL } \\
\text { ESPAÑOL }\end{array}$ & $\begin{array}{l}\text { CONCEPTOS ASOCIADOS/FUENTES } \\
\text { ASOCIADAS (EN INGLÉS) }\end{array}$ \\
\hline social relations (SR) & relaciones sociales (RS) & LCT, Specialization \\
\hline space grammar & gramática espacial & Stenglin (2008) \\
\hline specialisation code & código de especialización & LCT, Specialization \\
\hline specialised language & lenguaje especializado & Bernstein \\
\hline specialised syntax & sintaxis especializada & SFL, mass, technicality \\
\hline $\begin{array}{l}\text { specialist (consolidated) } \\
\text { word, [a] specialist } \\
\text { consolidated }\end{array}$ & $\begin{array}{l}\text { (sust.) palabra } \\
\text { (consolidada) especialista }\end{array}$ & $\begin{array}{l}\text { LCT, Semantics, semantic density, ESD, } \\
\text { wording tool, everydays }\end{array}$ \\
\hline Specialization & Especialización & LCT, dimension \\
\hline specialization code & código de especialización & LCT, Specialization \\
\hline specialization plane & plano de especialización & LCT, Specialization \\
\hline specialized discourse & discurso especializado & SFL \\
\hline specialized field & campo especializado & SFL, register, types of field \\
\hline specificity & especificidad & LCT, Semantics, semantic density \\
\hline stage & etapa & SFL, discourse semantics \\
\hline $\begin{array}{l}\text { strong/weak external } \\
\text { grammar }\end{array}$ & $\begin{array}{l}\text { gramática externa fuerte/ } \\
\text { débil }\end{array}$ & Bernstein* \\
\hline $\begin{array}{l}\text { strong/weak internal } \\
\text { grammar }\end{array}$ & $\begin{array}{l}\text { gramática interna fuerte/ } \\
\text { débil }\end{array}$ & Bernstein* \\
\hline $\begin{array}{l}\text { strength of semantic } \\
\text { density }\end{array}$ & $\begin{array}{l}\text { fortaleza de (la) densidad } \\
\text { semántica }\end{array}$ & LCT, Semantics, semantic density \\
\hline strengthening & $\begin{array}{l}\text { (sust.) fortalecimiento; } \\
\text { (vb.) fortalecer }\end{array}$ & LCT \\
\hline $\begin{array}{l}\text { strengths of epistemic- } \\
\text { semantic density }\end{array}$ & $\begin{array}{l}\text { fortalezas de densidad } \\
\text { semántica epistémica }\end{array}$ & LCT, Semantics, semantic density \\
\hline $\begin{array}{l}\text { stronger/weaker } \\
\text { semantic density (SD+/-) }\end{array}$ & $\begin{array}{l}\text { densidad semántica más } \\
\text { fuerte/más débil (DS+/-) }\end{array}$ & LCT, Semantics, semantic density \\
\hline subjectivist doxa & doxa subjetivista & LCT \\
\hline $\begin{array}{l}\text { subsumptive (cumulative) } \\
\text { sequencing }\end{array}$ & $\begin{array}{l}\text { secuenciamiento } \\
\text { (acumulativo) subsuntivo }\end{array}$ & $\begin{array}{l}\text { LCT, Semantics, semantic density, } \\
\text { degree of epistemological } \\
\text { condensation, sequencing analysis }\end{array}$ \\
\hline subtyping & subtipificación & $\begin{array}{l}\text { LCT, Semantics, semantic density, } \\
\text { degree of epistemological } \\
\text { condensation, clausing analysis }\end{array}$ \\
\hline
\end{tabular}




\begin{tabular}{|c|c|c|}
\hline TÉRMINO EN INGLÉS & $\begin{array}{l}\text { TRADUCCIÓN AL } \\
\text { ESPAÑOL }\end{array}$ & $\begin{array}{l}\text { CONCEPTOS ASOCIADOS/FUENTES } \\
\text { ASOCIADAS (EN INGLÉS) }\end{array}$ \\
\hline syndrome & síndrome & SFL \\
\hline system network & red sistémica & SFL, axis \\
\hline tacit taxonomy & taxonomía tácita & $\begin{array}{l}\text { LCT, Semantics, semantic density, ESD, } \\
\text { technicals }\end{array}$ \\
\hline Task (phase) & Tarea (, fase de) & $\mathrm{SFL}, \mathrm{R} 2 \mathrm{~L}$ \\
\hline taxonomic relations & relaciones taxonómicas & SFL, discourse semantics, IDEATION \\
\hline taxonomizing & $\begin{array}{l}\text { (sust.) taxonomización; } \\
\text { (vb.) taxonomizar }\end{array}$ & $\begin{array}{l}\text { LCT, Semantics, semantic density, } \\
\text { degree of epistemological } \\
\text { condensation, clausing analysis }\end{array}$ \\
\hline taxonomy & taxonomía & SFL \\
\hline $\begin{array}{l}\text { teaching/learning } \\
\text { practice }\end{array}$ & $\begin{array}{l}\text { práctica de enseñanza- } \\
\text { aprendizaje }\end{array}$ & $S F L, R 2 L$ \\
\hline technical discourse & discurso especializado & SFL, mass, technicality; Martin (1993) \\
\hline technical term & término técnico & SFL, axis, system network \\
\hline $\begin{array}{l}\text { technical word, [a] } \\
\text { technical }\end{array}$ & (sust.) palabra técnica & $\begin{array}{l}\text { LCT, Semantics, semantic density, ESD, } \\
\text { wording tool }\end{array}$ \\
\hline technicality & tecnicalidad & $\begin{array}{l}\text { SFL, type of mass, ideational } \\
\text { metafunction }\end{array}$ \\
\hline Temporality & Temporalidad & LCT, dimension \\
\hline tenor & tenor & SFL, register \\
\hline text reference & referencia textual & SFL, cohesion, reference \\
\hline textual aggregation & agregación textual & SFL, mass, aggregation \\
\hline topology & topología & SFL \\
\hline tracking move & $\begin{array}{l}\text { movimiento (interactivo) } \\
\text { de seguimiento }\end{array}$ & $\begin{array}{l}\text { SFL, discourse semantics, NEGOTIATION, } \\
\text { mass, aggregation; Martin (1992) }\end{array}$ \\
\hline translation device & dispositivo de traducción & $\mathrm{LCT}$ \\
\hline turn-taking resources & $\begin{array}{l}\text { recursos de toma de } \\
\text { turnos }\end{array}$ & $\begin{array}{l}\text { SFL, discourse semantics, NEGOTIATION, } \\
\text { mass, aggregation }\end{array}$ \\
\hline typology & tipología & SFL \\
\hline uncommon sense & $\begin{array}{l}\text { alejado del sentido } \\
\text { común, que escapa al } \\
\text { sentido común, fuera del } \\
\text { sentido común }\end{array}$ & SFL, code theory, Bernstein* \\
\hline
\end{tabular}




\begin{tabular}{|c|c|c|}
\hline TÉRMINO EN INGLÉS & $\begin{array}{l}\text { TRADUCCIÓN AL } \\
\text { ESPAÑOL }\end{array}$ & $\begin{array}{l}\text { CONCEPTOS ASOCIADOS/FUENTES } \\
\text { ASOCIADAS (EN INGLÉS) }\end{array}$ \\
\hline $\begin{array}{l}\text { uncommon sense } \\
\text { discourse }\end{array}$ & $\begin{array}{l}\text { discurso alejado del } \\
\text { sentido común }\end{array}$ & SFL, mass, technicality; Martin (1990) \\
\hline uncommon sense field & $\begin{array}{l}\text { campo alejado del } \\
\text { sentido común }\end{array}$ & SFL, register, field \\
\hline $\begin{array}{l}\text { vertical (cumulative) } \\
\text { sequencing }\end{array}$ & $\begin{array}{l}\text { secuenciamiento } \\
\text { (acumulativo) vertical }\end{array}$ & $\begin{array}{l}\text { LCT, Semantics, semantic density, } \\
\text { degree of epistemological } \\
\text { condensation, sequencing analysis }\end{array}$ \\
\hline vertical discourse & discurso vertical & Bernstein* \\
\hline verticality & verticalidad & Muller (2007) \\
\hline warring triangles & triángulos en pugna & Wignell \\
\hline weakening & $\begin{array}{l}\text { (sust.) debilitamiento; } \\
\text { (vb.) debilitar }\end{array}$ & LCT \\
\hline weakly classified & $\begin{array}{l}\text { de clasificación débil, } \\
\text { clasificado débilmente }\end{array}$ & Bernstein* \\
\hline word proxy, proxy word & $\begin{array}{l}\text { palabra sustitutiva, } \\
\text { sustitutivo de palabra }\end{array}$ & $\begin{array}{l}\text { LCT, Semantics, semantic density, ESD, } \\
\text { wording }\end{array}$ \\
\hline word-group proxy & $\begin{array}{l}\text { sustitutivo de } \\
\text { agrupamiento de } \\
\text { palabras }\end{array}$ & $\begin{array}{l}\text { LCT, Semantics, semantic density, ESD, } \\
\text { word-grouping }\end{array}$ \\
\hline word-grouping (tool) & $\begin{array}{l}\text { agrupamiento de palabras } \\
\text { (, herramienta de) }\end{array}$ & LCT, Semantics, semantic density, ESD \\
\hline wording (tool) & $\begin{array}{l}\text { empalabramiento } \\
\text { (, herramienta de) }\end{array}$ & LCT, Semantics, semantic density, ESD \\
\hline gravitation & gravitación & LCT, Semantics, semantic gravity \\
\hline worldly code & código mundano & $\begin{array}{l}\text { LCT, Semantics, semantic density, } \\
\text { semantic gravity }\end{array}$ \\
\hline
\end{tabular}

\section{Siglas y abreviaturas empleadas}

EC: epistemological condensation = condensación epistemológica (EC)

ESD: epistemic-semantic density = densidad semántica epistémica (DSE)

LCT: Legitimation Code Theory = Teoría de Códigos de Legitimación (TCL)

SFL: Systemic Functional Linguistics = Lingüística Sistémico Funcional (LSF)

R2L: Reading to Learn = Leer para aprender (Rose \& Martin, 2012)

vb.: verbo

sust.: sustantivo 


\section{Obras citadas}

DoRAn, Y. J., 2010: Knowledge and multisemiosis in undergraduate physics. Tesis para optar al grado de Bachelor of Liberal Studies, University of Sydney.

Doran, Y. J., 2015: Knowledge in physics through mathematics, image and language. Tesis doctoral, University of Sydney.

Halliday, M. A. K. \& C. M. I. M. Matthiessen, 1999: Construing experience through meaning: a language-based approach to cognition, Londres: Continuum.

HAO, J., 2015: Construing biology: an ideational perspective. Tesis doctoral, University of Sydney.

MARTIN, J. R., 1990: “Literacy in science: learning to handle text as technology” en Frances Christie(ed.): Literacy for a changing world, Melbourne: Australian Council for Educational Research, 79-117. MARTIN, J. R., 1992: English text: system and structure, Ámsterdam: John Benjamins.

MARTIN, J. R., 1993: "Technicality and abstraction: language for the creation of specialized texts" en M. A. K. Halliday \& J. R. Martin (eds.): Writing science: literacy and discursive power, Londres: Taylor and Francis, 223-241.

Maton, K., 2014: Knowledge and knowers: towards a realist sociology of education, Londres: Routledge.

Maton, K., 2016: "Starting points: resources and architectural glossary" en K. Maton, S. Hood y S. SHAY (eds.): Knowledge-building: Educational studies in Legitimation Code Theory, Londres: Routledge, 233-243.

Muller, J., 2007: "On splitting hairs: hierarchy, knowledge and the school curriculum” en Frances Christie y J. R. Martin (eds.): Language, knowledge and pedagogy, Londres: Continuum, 65-86.

Pornton, C., 1990: Address and the semiotics of social relations: a systemic-functional account of address forms and practices in Australian English. Tesis doctoral, University of Sydney.

QuiRoz, B., 2016: “Convenciones de notación sistémica”, Onomázein 33(2), 412-426.

ROSE, D. y J. R. MARTIN, 2012: Learning to write, reading to learn: genre, knowledge and pedagogy in the Sydney School, Londres: Equinox.

Stenglin, M., 2008: "Olympism: how a bonding icon gets its 'charge”" en L. Unsworth (ed.): Multimodal Semiotics: functional analysis in the contexts of education, Londres: Continuum, 50-66.

TANn, K., 2012: "The language of identity discourse: introducing a framework for functional iconography", Linguistics and the Human Sciences 8(3), 361-391. 